\title{
Coloproctologie pédiatrique : avancées récentes Introduction
}

\section{G. Podevin · G. Meurette}

(C) Lavoisier SAS 2015

Au même titre que la pathologie adulte, la pédiatrie a aussi son lot d'affections colorectales et proctologiques. Or l'exercice médical est souvent cloisonné entre les pédiatres et médecins adultes et le partage d'expérience est difficile. Pour autant, instaurer un dialogue est enrichissant pour tous les médecins et chirurgiens qui s'intéressent à la coloproctologie : il permet d'abord aux médecins adultes de connaître des affections (acquises ou malformatives) pour lesquelles de nombreuses spécificités liées à l'âge sont à prendre en compte. Il permet en retour aux pédiatres de connaître l'avenir des jeunes patients qu'ils soignent une fois l'âge adulte atteint.

L'objectif du dossier thématique qui suit est donc double : dresser un spectre des affections courantes en pédiatrie en précisant leur prise en charge actuelle, et en second lieu, présenter les avancées récentes dans la compréhension et la prise en charge des affections colorectales congénitales depuis l'enfance jusqu'à l'âge adulte.

C'est au travers de 5 chapitres que nous parcourrons cet éventail : les principaux symptômes proctologiques pédiatriques en pratique courante, la constipation de l'enfant, la maladie de Hirschsprung, le syndrome de Currarino et le devenir physique, social et professionnel des malformations colorectales pédiatriques (exemple de la maladie de Hirschsprung et les malformations anorectales).

En vous souhaitant une lecture agréable. 